\title{
Clinical significance of epigenetic silencing and re-expression of O6-methylguanine-DNA methyltransferase using epigenetic agents in laryngeal carcinoma
}

\author{
JING YANG $^{1}$, XIN-BING ZHU ${ }^{2}$, LI-XIA HE ${ }^{3}$, ZHAO-WEI GU ${ }^{1}$, MING-ZHU JIN ${ }^{1}$ and WEN-YUE JI ${ }^{1}$ \\ ${ }^{1}$ Department of Otorhinolaryngology, Shengjing Hospital of China Medical University, Shenyang, Liaoning 110004; \\ ${ }^{2}$ Department of General Sugery, General Hospital of Liaohe Oil Field, Panjin, Liaoning 124010; \\ ${ }^{3}$ Department of Otorhinolaryngology, Fushun Second Hospital, Fushun, Liaoning 113001, P.R. China
}

Received April 9, 2014; Accepted September 16, 2014

DOI: $10.3892 / 01.2014 .2662$

\begin{abstract}
The aim of the present study was to investigate the association between O6-methylguanine-DNA methyltransferase (MGMT) gene expression levels, and DNA methylation status and histone modifications in laryngeal squamous cell carcinoma (LSCC). Chromatin immunoprecipitation, methylation-specific polymerase chain reaction (PCR), and reverse transcription-quantitative PCR were performed to analyze histone modifications, DNA methylation status and mRNA expression levels in the promoter region of the MGMT gene in laryngeal carcinoma HEp-2 cells, as well as in 50 paired healthy and LSCC tissue samples. The present study demonstrated that treatment of HEp-2 cells with 5-aza-2'-deoxycytidine (Aza), a DNA methyltransferase inhibitor, significantly upregulated MGMT mRNA expression levels, reduced MGMT DNA methylation, reduced MGMT histone H3 lysine 9 (H3K9) di-methylation, and increased MGMT histone $\mathrm{H} 3$ lysine 4 di-methylation without a significant change in H3K9 acetylation. Trichostatin A (TSA), a histone deacetylase inhibitor, marginally upregulated MGMT mRNA expression levels without affecting the DNA methylation status, or H3K9 or H3K4 di-methylation, however, TSA treatment caused a significant increase in H3K9 acetylation. Furthermore, Aza and TSA combination treatment produced a synergistic effect. In the LSCC samples, the rate of DNA methylation in the MGMT gene was 54\%, compared with 24\% in the healthy control group $(\mathrm{P}<0.05)$. Therefore, data from
\end{abstract}

Correspondence to: Professor Wen-Yue Ji, Department of Otorhinolaryngology, Shengjing Hospital of China Medical University, 36 Sanhao Street, Shenyang, Liaoning 110004, P.R. China

E-mail: jiwenyuecmu2h@126.com

Key words: laryngeal carcinoma, O6-methylguanine-DNA methyltransferase gene, DNA methylation, histone modification, 5-aza-2'-deoxycytidine, trichostatin A the present study indicates that MGMT may serve as a novel therapeutic target in the treatment of LSCC.

\section{Introduction}

Laryngeal cancer is a common malignancy in otolaryngology, accounting for $1-5 \%$ of all cases of cancer, worldwide. It is the eleventh most common type of cancer, accounts for $35.4 \%$ of cases of head and neck cancer, and is the third most common type of head and neck malignancy, worldwide (1). O6-methylguanine-DNA methyltransferase (MGMT) is a key enzyme in the DNA repair network that removes mutagenic and cytotoxic adducts from O6-guanine in the DNA. Numerous carcinogens target O6-guanine, thus, the loss of MGMT gene expression results in the accumulation of unrepaired DNA damage and subsequent tumor development. MGMT is transcriptionally downregulated via the hypermethylation of $\mathrm{CpG}$ islands in its promoter region $(2,3)$.

The average level of MGMT mRNA expression is significantly lower in cancerous mucosa compared with the corresponding non-cancerous mucosa. Histone modification is closely associated with the DNA methylation status of a gene and is key for gene regulation. DNA hypermethylation in the promoter $\mathrm{CpG}$ islands of tumor suppressor genes (TSGs) inhibits transcriptional initiation and results in permanent gene silencing, a key process in carcinogenesis (4-6). Histone H3 lysine 9 (H3K9) acetylation and histone H3 lysine 4 (H3K4) di-methylation are associated with active gene transcription, however, H3K9 di-methylation is associated with gene repression $(7,8)$. Studies investigating the interaction between DNA methylation status and various histone modifications are currently ongoing.

To the best of our knowledge, no studies investigating the pattern of histone modifications in the TSG, MGMT in laryngeal carcinoma have been conducted. To establish a possible function for such epigenetic modifications of the MGMT gene in laryngeal carcinogenesis, the present report analyzed MGMT mRNA expression levels, DNA methylation status, and the levels of promoter region di-methyl-H3K9 (H3K9me2), H3K4me2 and acetyl-H3K9 (H3K9ac) following DNA methyltransferase inhibitor 5-aza-2'-deoxycytidine (Aza) and/or 
trichostatin A (TSA) treatment of laryngeal carcinoma HEp-2 cells. Furthermore, methylation-specific polymerase chain reaction (MSP) and reverse transcription (RT)-quantitative polymerase chain reaction (qPCR) were used to detect the association between MGMT gene expression levels and DNA methylation status in laryngeal squamous cell carcinoma (LSCC) tissues. Thus, the current report presents a mechanism for the inactivation of the TSG, MGMT in LSCC tissues.

\section{Materials and methods}

Cell line and tissue samples. HEp-2 cells were cultured in RPMI-1640 medium (pH 7.2; Gibco BRL, Life Technologies Inc., Grand Island, NY, USA) supplemented with $10 \%$ fetal bovine serum (inactivated under $56^{\circ} \mathrm{C}$ for $30 \mathrm{~min}$ ), $100 \times 10^{3} \mathrm{U} / 1$ penicillin and $100 \times 10^{3} \mathrm{U} / 1$ streptomycin, and were cultured in a closed incubator in a 5\% humidified $\mathrm{CO}_{2}$ atmosphere at a constant temperature of $37^{\circ} \mathrm{C}$. Cells were required to reach the logarithmic growth phase and a viable cell count of $95-100 \%$ immediately prior to the experiments.

Fifty LSCC patients, who were diagnosed and treated between January 2008 and May 2009 at the Shengjing Hospital of China Medical University (Shenyang, China), were evaluated in the present study. Prior to surgery, the patients were pathologically diagnosed with LSCC; however, no chemotherapy or radiation was administered. Control mucosa samples were obtained from the patients who had received a total laryngectomy; the samples were obtained from tissue $>2.0 \mathrm{~cm}$ from the tumor margin. Field cancerization may result in the tumor affecting a larger tissue area; therefore, only histologically healthy control mucosa samples were used in the present study. The samples were immediately snap-frozen in liquid nitrogen and stored at $-80^{\circ} \mathrm{C}$. Relevant clinical characteristics of the patients (age, gender, state of nodal metastases, and clinical stage, $T$ stage and differentiation grade of the tumor) were extracted from the patients' files. Tumor staging was conducted according to the International Union against Cancer 2002 tumor node metastasis classification (9). The patient tumor samples were collected following receipt of informed consent.

Treatment of cells with Aza and TSA. HEp-2 cells were divided into three groups: (i) Aza group, $5 \mu \mathrm{mol} / 1 \mathrm{Aza}$ (Sigma-Aldrich, St. Louis, MO, USA) was added and cultured for $72 \mathrm{~h}$; (ii) TSA group, $300 \mathrm{nmol} / \mathrm{l} \mathrm{TSA}$ (Sigma-Aldrich) was added and cultured for $24 \mathrm{~h}$; and (iii) Aza plus TSA group, $5 \mu \mathrm{mol} / \mathrm{l}$ Aza was added and cultured for $48 \mathrm{~h}$ prior to adding $300 \mathrm{nmol} / \mathrm{l} \mathrm{TSA}$ and continuing to culture for $24 \mathrm{~h}$. Control cells of the same batch, were not treated with any agent. The dose, time period and sequence of Aza and/or TSA treatment were based on similar preliminary studies (10-12).

$R N A$ extraction and RT-qPCR. Total RNA was isolated from cultured cells and tissues using TRIzol ${ }^{\circledR}$ reagent (Invitrogen Life Technologies, Carlsbad, CA, USA) according to the manufacturer's instructions. Total RNA treated with $2 \mu \mathrm{g}$ DNase I (Thermo Fisher Scientific, Pittsburgh, PA, USA) was converted to complementary (c)DNA using a Reverse Transcription System kit (Thermo Fisher Scientific). RNA was excluded from the cDNA synthesis reactions and served
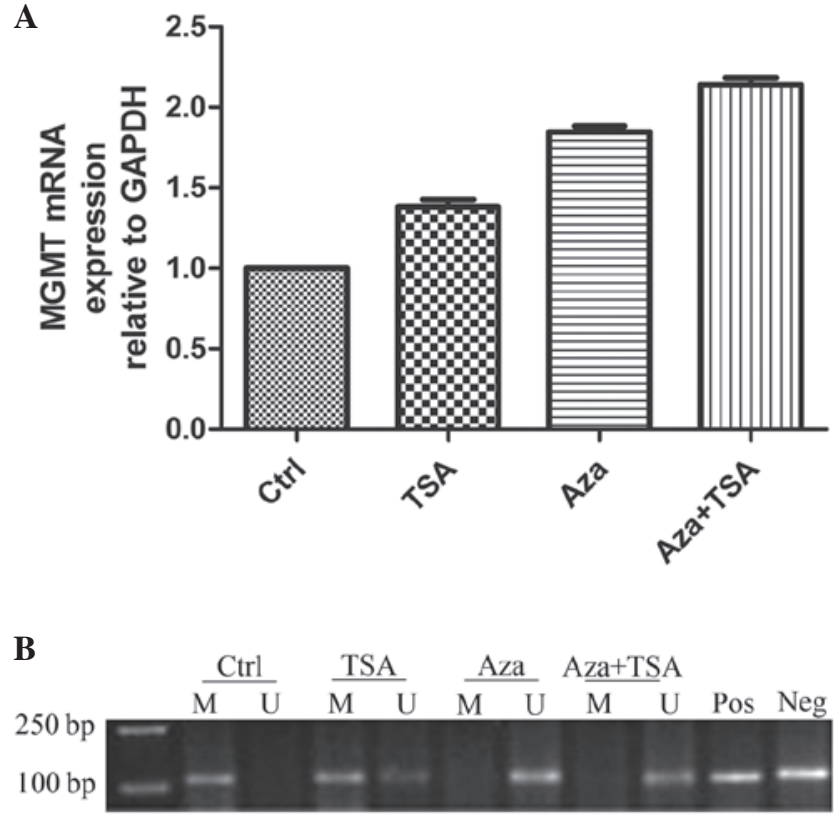

Figure 1. MGMT mRNA expression levels and DNA methylation status in laryngeal carcinoma HEp-2 cells before and after treatments with Aza and TSA. (A) RT-qPCR analysis of MGMT mRNA expression in laryngeal carcinoma HEp-2 before (Ctrl) and after treatment with Aza and/or TSA. (B) Methylation-specific PCR analysis of DNA methylation at the MGMT promoter region in laryngeal carcinoma HEp-2 cells before and after treatment with Aza and/or TSA. Lane M, methylated alleles (121 bp); lane U, unmethylated alleles (122 bp); Pos, positive control; Neg, negative control. A minimum of three independent experiments were performed, which all produced similar results. MGMT, O6-methylguanine-DNA methyltransferase; Ctrl, control; TSA, trichostatin A; Aza, 5-aza-2'-deoxycytidine; RT-qPCR, reverse transcription-quantitative polymerase chain reaction.

as a negative control. PCR was performed in a $25-\mu 1$ reaction volume using the Maxima SYBR ${ }^{\circledR}$ Green/ROX qPCR Master Mix (Thermo Fisher Scientific) under the following conditions: $95^{\circ} \mathrm{C}$ for $10 \mathrm{~min}$, followed by 40 cycles of $95^{\circ} \mathrm{C}$ for $30 \mathrm{sec}$ (denaturation), $56^{\circ} \mathrm{C}$ for $30 \mathrm{sec}$ (annealing), and $72^{\circ} \mathrm{C}$ for $30 \mathrm{sec}$ (extension); and an additional extension of $72^{\circ} \mathrm{C}$ for $10 \mathrm{~min}$. The PCR product length for the MGMT gene fragment was $151 \mathrm{bp}$ and the MGMT primers were as follows: Upstream, 5'-CGAAATAAAGCTCCTGGGCA-3' and downstream, 5'-GAACTCTTCGATAGCCTCGGG-3'. The PCR product length for the GAPDH gene fragment was 115 bp and the GAPDH primers were as follows: Upstream, 5'-TCCCATCACCATCTTCCAG-3' and downstream, 5'-ATGAGTCCTTCCACGATACC-3'. The $2^{-\Delta \Delta c t}$ method was used to calculate the MGMT gene expression levels relative to an internal GAPDH control and, thus, the fold changes of the gene expression levels. All experiments were repeated three times for statistical analysis.

MSP. Genomic DNA was prepared from cell lines and tissues using the phenol/chloroform extraction protocol and was modified by bisulfite treatment. Briefly, DNA was denatured by incubating with $0.3 \mathrm{~mol} / 1 \mathrm{NaOH}$ at $37^{\circ} \mathrm{C}$ for $30 \mathrm{~min}$, followed by incubation with $10 \mathrm{mmol} / \mathrm{l}$ hydroquinone and $3 \mathrm{~mol} / \mathrm{l}$ sodium bisulfite (Sigma-Aldrich) at $55^{\circ} \mathrm{C}$ for $16 \mathrm{~h}$. Modified DNA was purified using the Wizard DNA Clean-Up System (Promega Corporation, Madison, WI, USA) according to the manufacturer's instructions. The MGMT PCR product 
$\mathbf{A}$

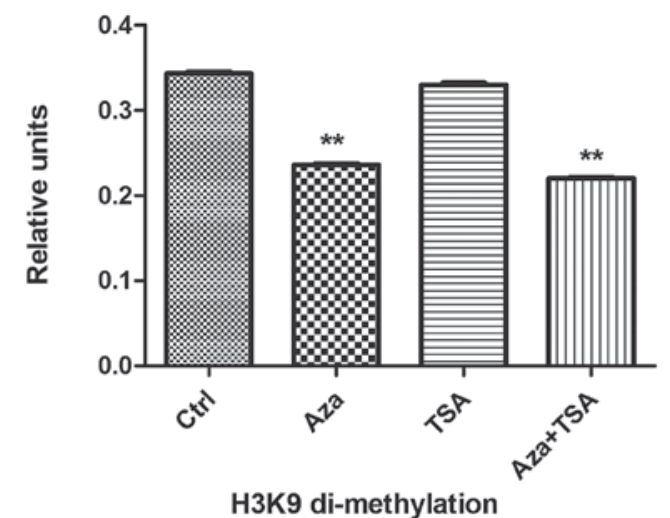

B

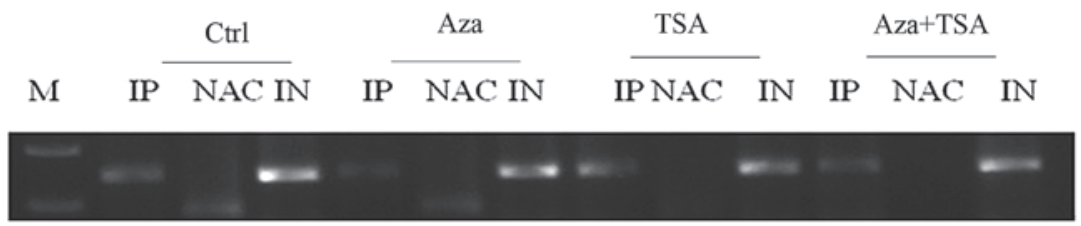

Figure 2. ChIP analysis of H3K9 di-methylation before and after treatment of HEp-2 cells with Aza, TSA, or Aza and TSA. Three independent ChIP assays were performed using an antibody that recognizes di-methyl H3K9 at the O6-methylguanine-DNA methyltransferase promoter region. (A) Summary of PCR analyses of ChIP assays. The mean precipitated DNA/input DNA ratios demonstrated on the y-axis represent the relative values of H3K9 di-methylation. Mean H3K9 di-methylation levels are demonstrated by the standard error bars and ${ }^{* *} \mathrm{P}<0.01$. (B) PCR assay. Ctrl, prior to treatment; Aza, following treatment with Aza; TSA, following treatment with TSA; Asa + TSA, following treatment with Aza and TSA. ChIP, chromatin immunoprecipitation; H3K9, histone 3 lysine 9; Aza, 5-aza-2'-deoxycytidine; TSA, trichostatin A; PCR, polymerase chain reaction; IP, immunoprecipitated DNA; NAC, no antibody control; IN, input DNA from whole cell lysate.

A

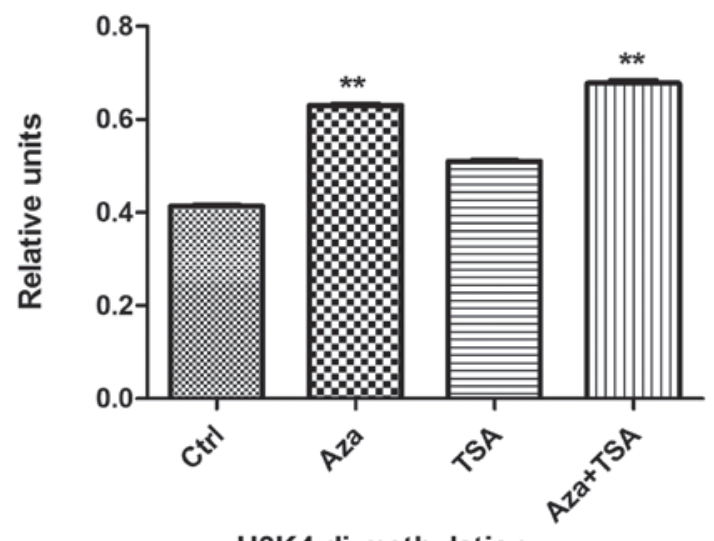

B

H3K4 di-methylation

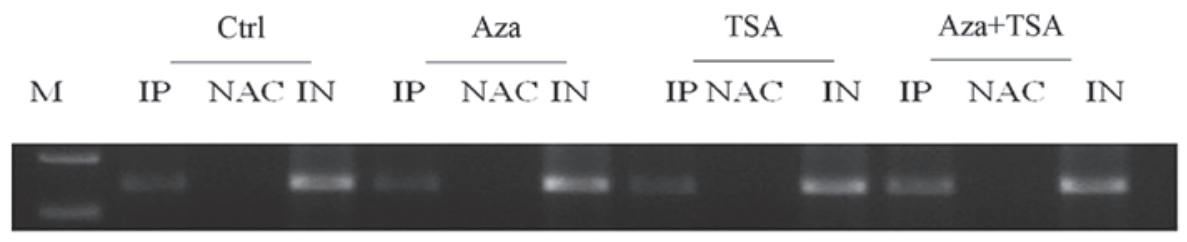

Figure 3. ChIP analysis of H3K4 di-methylation before and after treatment of HEp-2 cells with Aza, TSA, or Aza and TSA. Three independent ChIP assays were performed using an antibody that recognizes di-methyl H3K4 at the O6-methylguanine-DNA methyltransferase promoter region. (A) Summary of PCR analyses of ChIP assays. The mean precipitated DNA/input DNA ratios demonstrated on the y-axis represent the relative values of H3K4 di-methylation. Mean H3K4 di-methylation levels are demonstrated by the standard error bars and ${ }^{* *} \mathrm{P}<0.01$ vs. control group. (B) PCR assay. Ctrl, prior to treatment; Asa, following treatment with Aza; TSA, following treatment with TSA; Asa + TSA, following treatment with Aza and TSA. ChIP, chromatin immunoprecipitation; H3K4, histone 3 lysine 4; Aza, 5-aza-2'-deoxycytidine; TSA, trichostatin A; PCR, polymerase chain reaction; IP, immunoprecipitated DNA; NAC, no antibody control; IN, input DNA from whole cell lysate.

length was $121 \mathrm{bp}$ for the methylated fragment and $122 \mathrm{bp}$ for the unmethylated fragment. The MSP primers are located in the promoter region of the MGMT gene and were as follows: Upstream,5'-GGTCGTTTGTACGTTCGC-3'anddownstream, 
A

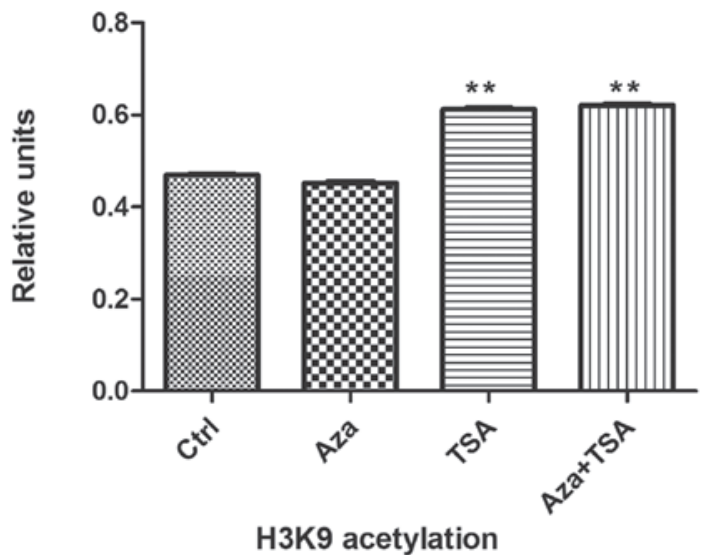

B

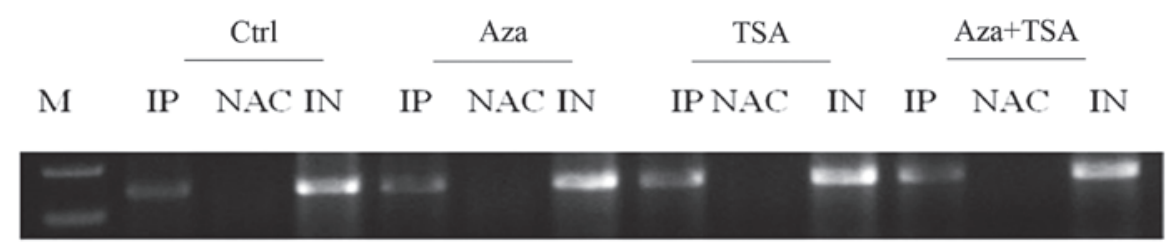

Figure 4. ChIP analysis of H3K9 acetylation before and after treatment of HEp-2 cells with Aza, TSA, or Aza and TSA. Three independent ChIP assays were performed using an antibody that recognizes H3K9 acetylation at the O6-methylguanine-DNA methyltransferase promoter region. (A) Summary of PCR analyses of ChIP assays. The mean precipitated DNA/inputDNA ratios demonstrated on the y-axis represent the relative values of H3K9 acetylation. Mean H3K9 acetylation levels are demonstrated by the standard error bars and ${ }^{* *} \mathrm{P}<0.05$ vs. control group. (B) PCR assay. Ctrl, prior to treatment; Asa following treatment with Aza; TSA, following treatment with TSA; Asa + TSA, following treatment with Aza and TSA. ChIP, chromatin immunoprecipitation; H3K9, histone 3 lysine 9; Aza, 5-aza-2'-deoxycytidine; TSA, trichostatin A; PCR, polymerase chain reaction; IP, immunoprecipitated DNA; NAC, no antibody control; IN, input DNA from whole cell lysate..

5'-GACCGATACAAACCGAACG-3' for the methylation primers; and upstream, 5'-GTAGGTTGTTTGTATGTTTGT-3' and downstream, 5'-AACCAATACAAACCAAACA-3' for the unmethylated primers. PCR was performed under the following conditions: $95^{\circ} \mathrm{C}$ for $12 \mathrm{~min}$, followed by 32 cycles of $95^{\circ} \mathrm{C}$ for $30 \mathrm{sec}$ (denaturation), $61^{\circ} \mathrm{C}$ for $45 \mathrm{sec}$ (annealing) and $72^{\circ} \mathrm{C}$ for $30 \mathrm{sec}$ (extension). An additional extension of $72^{\circ} \mathrm{C}$ for 7 min was performed prior to agarose gel electrophoresis and ethidium bromide staining. A Chemilmanger 5500 automatic image analyzer (Alpha Innotech Corporation, San Leandro, CA, USA) was used to obtain imaging data and analyze the electrophoresis results. All experiments were repeated three times for statistical analysis.

Chromatin immunoprecipitation assay (ChIP). ChIP was performed as previously described with specific modifications (13). Briefly, $\sim 1.75 \times 10^{7} \mathrm{HEp}-2$ cells, treated as described above, were fixed with $1 \%$ formaldehyde at $37^{\circ} \mathrm{C}$ for $20 \mathrm{~min}$, resuspended in lysis buffer ( $1 \%$ sodium dodecyl sulfate, $10 \mathrm{mmol} / \mathrm{l}$ EDTA, $50 \mathrm{mmol} / \mathrm{l}$ Tris $\mathrm{HCl} ; \mathrm{pH}$ 8.1) and sonicated to generate $\sim 500$-bp DNA fragments. Antibodies against H3K9me2, H3K4me2 or H3K9ac (Upstate Biotechnology Inc., Lake Placid, NY, USA) were used to immunoprecipitate the major soluble chromatin fraction. The remaining soluble fraction was incubated with normal rabbit IgG (negative control) and used as a DNA input control. DNA-protein crosslinks were reversed by heating the samples to $65^{\circ} \mathrm{C}$ for $5 \mathrm{~h}$ and digesting with proteinase K. DNA was then extracted using the phenol/chloroform protocol. The ChIP experiments were repeated three times.

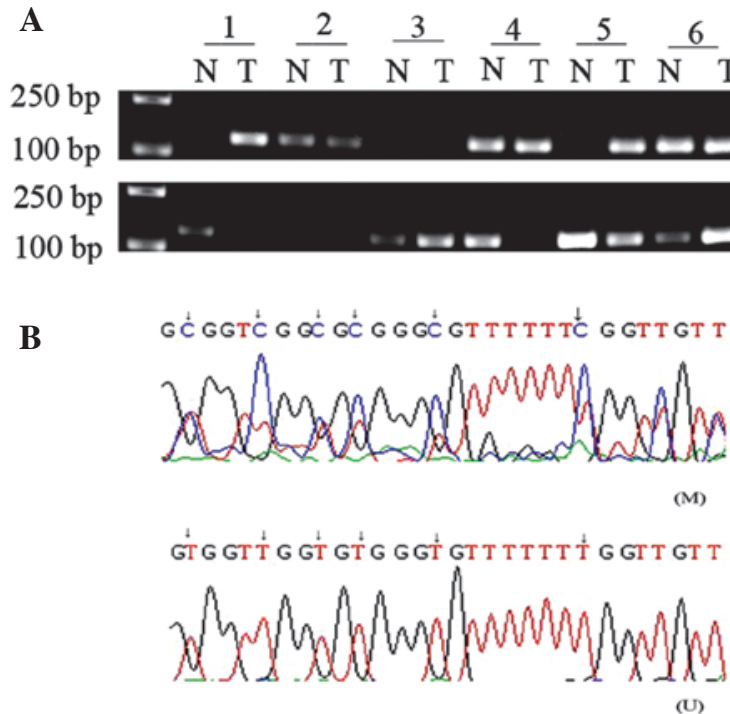

Figure 5. Methylation status of the MGMT gene promoter in laryngeal carcinoma tissues and the paired adjacent healthy tissues. (A) Methylation-specific polymerase chain reaction demonstrates the DNA methylation pattern of MGMT in laryngeal squamous cell carcinoma and the corresponding healthy tissue samples. 1-6, case sample number; lane N, non-malignant laryngeal tissue; lane T, laryngeal carcinoma tissues; M, methylation bands; U, unmethylated bands. (B) Demonstration of MGMT promoter methylation by sequencing following sodium bisulfite modification. MGMT, O6-methylguanine-DNA methyltransferase.

PCR analysis of the immunoprecipitated DNA. PCR reactions were performed using $2 \mu \mathrm{limmunoprecipitated} \mathrm{DNA,} \mathrm{a} \mathrm{negative}$ 


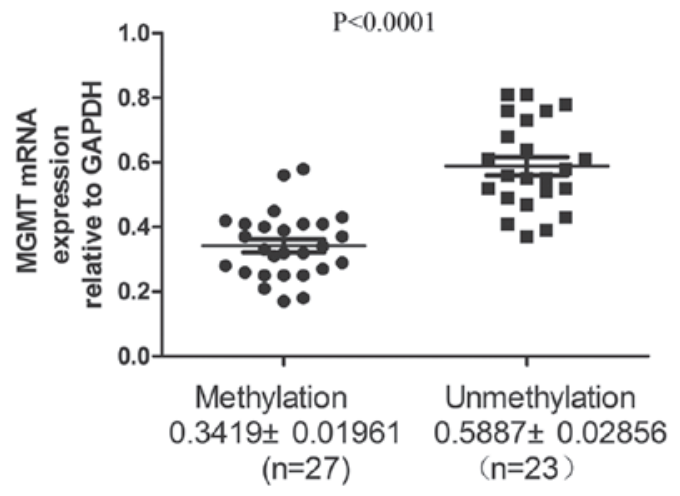

Figure 6.Reverse transcription-quantitative polymerase chain reaction analysis of MGMT mRNA expression levels in MGMT methylated and unmethulated laryngeal squamous cell carcinoma tissue. MGMT, O6-methylguanine-DNA methyltransferase.

control and a DNA input control. The PCR product length for the MGMT gene fragment was $171 \mathrm{bp}$ and the ChIP-PCR primers, located in the MGMT promoter region, were as follows: Upstream, 5'-CCCCATCTCCAAATAAGGTCA-3' and downstream, 5'-CCTAGACACTGCCAGAGCCTG-3'. PCR products were resolved on $2 \%$ agarose gels (Promega Corporation) and quantified using a GelDoc 1000 (Bio-Rad, Hercules, CA, USA) and Molecular Analyst software (Alpha Innotech Corporation). The levels of H3K9, and H3K4 di-methylation and H3K9 acetylation in each immunoprecipitate were determined by quantifying the intensities of the PCR products in the immunoprecipitated versus input DNA. ChIP was repeated a minimum of two times and three independent PCR analyses of each sample were performed.

Wound healing assay. HEp-2 cells were seeded in six-well plates and cultured in RPMI-1640 medium supplemented with $10 \%$ fetal bovine serum. A wound was created in the center of the cell monolayer using a sterile plastic pipette tip. The cells were cultured for $24 \mathrm{~h}$ to allow cell migration. To assess the ability of the cells to migrate into the wound area, an inverted microscope was used to capture images of the cells 0 and $24 \mathrm{~h}$ after wounding.

Matrigel ${ }^{\circledR}$ invasion assay. HEp-2 cells $\left(5 \times 10^{4}\right)$ cultured in $200 \mu \mathrm{l}$ serum-free RPMI-1640 medium were seeded onto the upper chambers of Matrigel ${ }^{\circledR}$-coated Transwell ${ }^{\circledR}$ filters (pore size, $8 \mu \mathrm{m}$; Corning Life Sciences, Corning, NY, USA). RPMI-1640 $(500 \mu \mathrm{l})$ supplemented with $10 \%$ fetal bovine serum was added to the lower chambers as a chemoattractant. Cells were incubated at $37^{\circ} \mathrm{C}$ in a humidified $5 \% \mathrm{CO}_{2}$ atmosphere for $24 \mathrm{~h}$. Cells that had successfully invaded through the inserts were fixed in $4 \%$ paraformaldehyde for $30 \mathrm{~min}$ and stained with methylrosanilinium chloride. The invaded cells were counted from five preselected microscopic fields (magnification, $\mathrm{x} 200$ ). The mean result of the assay was obtained from three independent experiments.

Statistical analysis. The ratio results were expressed as the mean \pm standard deviation. Student's t-test was used to calculate the significance between the treated and control samples. The Spearman rank-correlation test was used to examine the association between the MGMT expression level, and DNA hypermethylation and histone modification. Furthermore, $\chi^{2}$ and Fisher's exact tests were adopted to analyze the aberrant DNA hypermethylation of MGMT within the clinicopathological parameters. Statistical calculations were performed using SPSS version 13.0 (SPSS Inc., Chicago, IL, USA) and $\mathrm{P}<0.05$ was considered to indicate a statistically significant difference.

\section{Results}

Analysis of MGMT mRNA expression levels and DNA methylation status in laryngeal carcinoma HEp-2 cells before and after treatment with Aza and TSA. RT-qPCR was performed to assess the level of MGMT mRNA expression in the control group (no Aza or TSA treatment; relative mRNA expression level, 1) compared with the expression level following treatment with TSA (relative mRNA expression level, 1.383 $\pm 0.0417 ; \mathrm{P}<0.05$ ), Aza (relative mRNA expression level, $1.847 \pm 0.0 .03844 ; \mathrm{P}<0.01)$ and a combination of Aza and TSA (relative mRNA expression level, 2.140 \pm 0.04509 ; $\mathrm{P}<0.01$; Fig. 1A).

To identify whether DNA methylation was associated with the changes in MGMT mRNA expression level, the present study used MSP to assess the DNA methylation status of MGMT in HEp-2 cells. DNA methylation in the MGMT gene promoter region (presence of a methylation band only) was apparent prior to treatment. TSA treatment induced hemi-methylation of the MGMT promoter $\mathrm{CpG}$ islands (co-presence of methylation and non-methylation bands) and Aza treatment resulted in total DNA demethylation (presence of a methylation band only). The effect of Aza and TSA combination treatment was similar to that of Aza alone (Fig. 1B)

Differential effects of Aza and TSA treatment on histone modification in laryngeal carcinoma HEp-2 cells. To elucidate the effect of epigenetic agents on histone modifications, HEp-2 cells were treated with Aza and TSA. Aza treatment resulted in a significant decrease in the levels of $\mathrm{H} 3 \mathrm{~K} 9$ di-methylation in the MGMT promoter region $(0.343 \pm 0.003$ vs. $0.236 \pm 0.001 ; \mathrm{P}<0.01)$ and $\mathrm{TSA}$ treatment resulted in a marginal reduction in $\mathrm{H} 3 \mathrm{~K} 9$ di-methylation $(0.343 \pm 0.003$ vs. $0.330 \pm 0.003 ; \mathrm{P}>0.05)$. The effect of combined Aza and TSA treatment on $\mathrm{H} 3 \mathrm{~K} 9$ di-methylation was similar to that of Aza alone (0.343 \pm 0.003 vs. $0.220 \pm 0.001$; $\mathrm{P}<0.01$; Fig. 2). To analyze the level of $\mathrm{H} 3 \mathrm{~K} 4$ di-methylation at MGMT promoter regions, ChIP assays were performed on HEp-2 cells. Aza treatment significantly increased the level of H3K4 di-methylation at the MGMT promoter $(0.484 \pm 0.007$ vs. $0.631 \pm 0.002 ; \mathrm{P}<0.01)$; however, TSA treatment did not affect H3K4 di-methylation $(0.484 \pm 0.007$ vs. $0.510 \pm 0.002$; $\mathrm{P}>0.05$; Fig. 3). The effect of combined Aza and TSA treatment on the level of $\mathrm{H} 3 \mathrm{~K} 4$ di-methylation was similar to that of Aza alone $(0.484 \pm 0.007$ vs. $0.677 \pm 0.006 ; \mathrm{P}<0.01)$. Furthermore, H3K9 acetylation was significantly increased at MGMT promoter regions following treatment with TSA $(0.470 \pm 0.001$ vs. $0.612 \pm 0.003$; $\mathrm{P}<0.01)$, however, Aza exhibited no marked effect on H3K9 acetylation $(0.470 \pm 0.001$ vs. $0.452 \pm 0.002, \mathrm{P}>0.05)$. The effect of combined Aza and TSA 


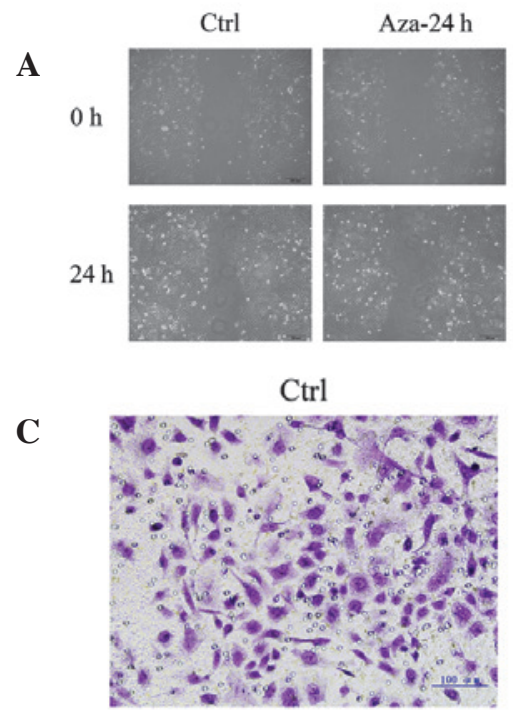

Aza-48h

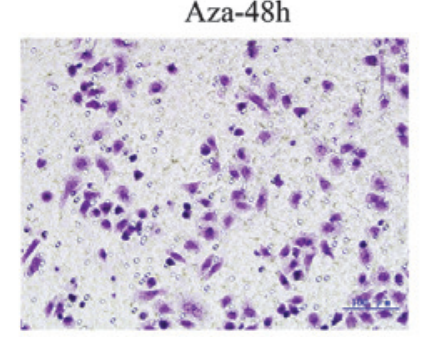

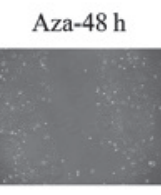
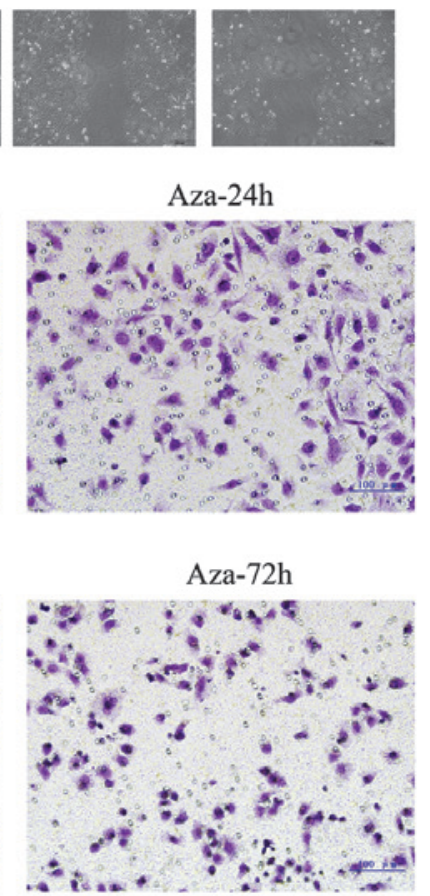

B

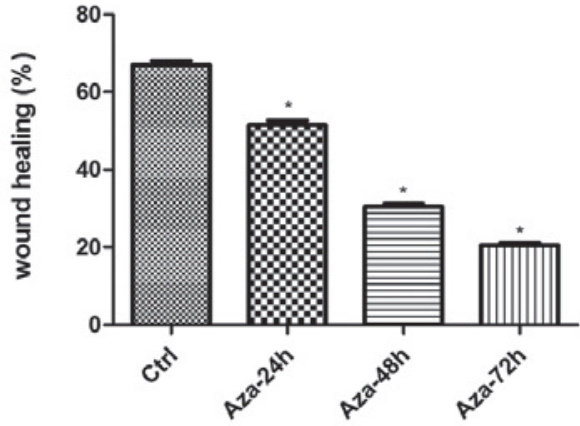

$\mathbf{D}$

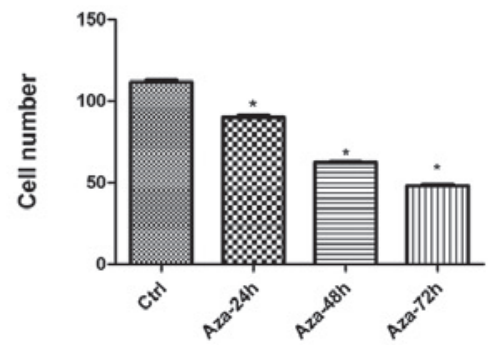

Figure 7. Effect of Aza on the migration and invasion of HEp-2 cells in vitro. (A) Wound healing assay of HEp-2 cells. Images were captured $24 \mathrm{~h}$ after wounding (magnification, x100). (B) Percentage wound closure was measured in at least three randomly selected regions (means \pm SD). (C) Invasion ability of HEp-2 cells was determined by a Matrigel ${ }^{\circledR}$ invasion assay following treatment with Aza. Representative images of treated and untreated cells are presented (magnification, x200). (D) Number of cells invading the wounded area at $24 \mathrm{~h}$ (means $\pm \mathrm{SD}$ ). "P<0.01 vs. Ctrl. Aza, 5-aza-2'-deoxycytidine; Ctrl, control; $\mathrm{SD}$, standard deviation.

treatment on $\mathrm{H} 3 \mathrm{~K} 9$ acetylation levels was similar to that of TSA alone $(0.470 \pm 0.0007$ vs. $0.621 \pm 0.0023, \mathrm{P}<0.05$; Fig. 4).

Frequent DNA methylation of MGMT in LSCC and its clinical significance. The methylation status of MGMT in LSCC tissue and paired adjacent healthy tissue samples was detected using MSP. The DNA methylation rate of MGMT was significantly higher in laryngeal cancer (54\%) compared with $24 \%$ in the healthy control group $(\mathrm{P}<0.05$; Fig. $5 \mathrm{~A}$; data not shown). The methylated and unmethylated products of MSP were sequenced, confirming that sodium bisulfite modification was sufficient for the DNA (Fig. 5B). The present study also identified that the DNA methylation status of MGMT exhibited no correlation with the age or gender of the patient, the degree of tumor differentiation, the tumor $\mathrm{T}$ stage or lymph node metastasis in LSCC tissues ( $\mathrm{P}>0.05$; Table I).

Low expression level of MGMT is associated with DNA methylation status in LSCC tissues. RT-qPCR was performed to assess the MGMT mRNA expression level in LSCC tissues. The present study identified that MGMT mRNA expression levels were significantly lower in LSCC tissues exhibiting MGMT DNA methylation compared with unmethylated DNA $(0.3419 \pm 0.01916$ vs. $0.5887 \pm 0.02856$; $P<0.0001$; Fig. 6 and Table II).
Migration and invasion inhibition of HEp-2 cells following treatment with Aza. To investigate the inhibitory effect of Aza on the migration and invasion of HEp-2 cells, a wound-healing and Matrigel ${ }^{\circledR}$ invasion assay was performed. The present study demonstrated that $24 \mathrm{~h}$ after establishing the wound, the control group achieved the most wound closure

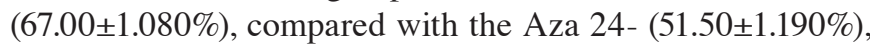
$48-(30.50 \pm 0.6455 \%)$ and $72-\mathrm{h}(20.50 \pm 0.6455 \%)$ groups $(\mathrm{F}=510.9 ; \mathrm{P}<0.0001$; Fig. 7A and $\mathrm{B})$. The Matrigel ${ }^{\circledR}$ invasion assay demonstrated that the number of invading HEp-2 cells was $111.5 \pm 1.190,89.75 \pm 1.250,62.25 \pm 0.4787$, and $48.00 \pm 0.9129 \%$ in the control, Aza 24-, 48- and 72-h groups, respectively. The number of invading HEp-2 cells was significantly reduced following Aza treatment when compared with the control group $(\mathrm{F}=794.5 ; \mathrm{P}<0.0001$; Fig. $7 \mathrm{C}$ and D). Thus, Aza significantly reduced HEp-2 cell migration and invasion.

\section{Discussion}

A growing number of TSGs are being identified that are inactivated by epigenetic, rather than classic, mutation/deletion events $(14,15)$. Unlike mutational inactivation, methylation is reversible, thus, demethylating agents and inhibitors of histone deacetylases (HDACs) have been evaluated in clinical trials (16-19). Aza, a pyrimidine analogue with the 
Table I. Clinicopathological parameters and MGMT methylation status of tissue samples from laryngeal squamous cell carcinoma patients.

\begin{tabular}{|c|c|c|c|c|}
\hline \multirow[b]{2}{*}{ Variable } & \multirow{2}{*}{$\begin{array}{l}\text { Patients, } \\
\mathrm{n}\end{array}$} & \multicolumn{2}{|c|}{$\begin{array}{l}\text { MGMT methylation } \\
\text { status, } \mathrm{n}(\%)\end{array}$} & \multirow[b]{2}{*}{ P-valuc } \\
\hline & & M & $\mathrm{U}$ & \\
\hline Gender & & & & 0.517 \\
\hline Male & 44 & $23(52.3)$ & $21(47.7)$ & \\
\hline Female & 6 & $4(66.7)$ & $2(33.3)$ & \\
\hline Age, years & & & & 0.162 \\
\hline$<60$ & 24 & $16(66.7)$ & $8(33.3)$ & \\
\hline$\geq 60$ & 26 & $11(42.3)$ & $15(57.7)$ & \\
\hline Tumor $\mathrm{T}$ stage & & & & 0.982 \\
\hline $\mathrm{T} 1+\mathrm{T} 2$ & 24 & $13(54.2)$ & $11(45.8)$ & \\
\hline $\mathrm{T} 3+\mathrm{T} 4$ & 26 & $14(53.8)$ & $12(46.2)$ & \\
\hline $\begin{array}{l}\text { Lymphatic } \\
\text { metastasis }\end{array}$ & & & & 0.968 \\
\hline Positive & 11 & $6(54.5)$ & $5(45.5)$ & \\
\hline Negative & 39 & $21(53.8)$ & $18(46.2)$ & \\
\hline Tumor Grade & & & & 0.586 \\
\hline Well-differentiated & 29 & $18(62.0)$ & $11(38.0)$ & \\
\hline $\begin{array}{l}\text { Moderately and } \\
\text { poorly differentiated }\end{array}$ & 21 & $9(42.9)$ & $12(57.1)$ & \\
\hline Type & & & & 0.747 \\
\hline Glottic & 23 & $13(56.5)$ & $10(43.5)$ & \\
\hline Supraglottic & 27 & $14(51.9)$ & $13(47.1)$ & \\
\hline
\end{tabular}

$\mathrm{P}<0.05$ was considered to indicate a statistically significant difference. The $\chi^{2}$ test was used to analyze the data. MGMT, O6-methylguanine-DNA methyltransferase; M, methylated and partially methylated; $\mathrm{U}$, unmethylated.

Table II. Association between MGMT mRNA expression levels and DNA methylation status in laryngeal squamous cell carcinoma tissues.

\begin{tabular}{lcc}
\hline $\begin{array}{l}\text { DNA methylation } \\
\text { status }\end{array}$ & Cases, $\mathrm{n}$ & $\begin{array}{l}\text { MGMT mRNA } \\
\text { expression level }\end{array}$ \\
\hline Methylated & 27 & $0.3419 \pm 0.01961$ \\
Unmethylated & 23 & $0.5887 \pm 0.02856$
\end{tabular}

The student's t-test was used to calculate the significance of differences in MGMT mRNA expression level between the methylated and unmethylated groups $(\mathrm{t}=17.628 ; \quad \mathrm{P}<0.0001)$ MGMT, O6-methylguanine-DNA methyltransferase.

2'-deoxycytidine fifth carbon atom replaced by a nitrogen atom, binds to DNA molecules during replication and subsequently forms a complex with DNA methyltransferase (DNMT1). This complex inhibits the transmethylation activity of DNMT1. TSA is a HDAC inhibitor that causes DNA histone hyperacetylation and subsequently induces p21 (WAF1/CIP1) gene expression. Upregulation of p21 (WAF1/CIP1) gene transcription may result in cell cycle arrest and inhibition of cell growth by regulating cell cycle regulatory factors and the expression levels of apoptosis-associated proteins $(17,20)$.

In the present study, it was identified that DNA hypermethylation-silenced MGMT in laryngeal carcinoma HEp-2 cells is characterized by $\mathrm{H} 3 \mathrm{~K} 9$ hypermethylation, and $\mathrm{H} 3 \mathrm{~K} 9$ and $\mathrm{H} 3 \mathrm{~K} 4$ hypomethylation at the promoter. Following treatment with Aza alone or in combination with TSA, H3K9 di-methylation decreased and $\mathrm{H} 3 \mathrm{~K} 4$ di-methylation increased at the promoter, consistent with DNA demethylation and reactivation of MGMT expression levels. In addition, the effect of combined Aza and TSA treatment on MGMT expression levels was stronger than that of Aza treatment alone. Treatment of laryngeal carcinoma HEp-2 cells with Aza appeared to reverse the MGMT gene methylation status and demethylate the promoter region. Furthermore, the effect of combined Aza and TSA treatment on MGMT expression levels was stronger than that of Aza treatment alone. In the present study, the MGMT methylation status was not affected by TSA alone as TSA is an HDAC inhibitor, which does not affect the gene promoter methylation status. The primary effect of epigenetic agents on MGMT gene expression levels was promoter hypermethylation: MGMT gene expression level was significantly upregulated by Aza, marginally upregulated by TSA, and synergistically upregulated using a combination of the two epigenetic agents. The results indicate that the primary epigenetic factor influencing gene expression levels and downregulating the TSG transcription was MGMT gene DNA methylation, however, histone modifications were also key. ChIP assays were conducted to investigate the association between the DNA methylation status and histone modifications. ChIP is a technique used to identify the presence of particular DNA-binding proteins that may modulate chromatin structure and/or transcriptional characteristics of the specific region of DNA with which the DNA-binding proteins are associated. The present study demonstrated that H3K9 di-methylation levels in the MGMT promoter region correlated with the DNA methylation status. MGMT reactivation by Aza was accompanied by DNA demethylation and a decrease in $\mathrm{H} 3 \mathrm{~K} 9$ di-methylation levels. In contrast to $\mathrm{H} 3 \mathrm{~K} 9$ di-methylation, $\mathrm{H} 3 \mathrm{~K} 4$ di-methylation in the promoter region was inversely correlated with the DNA methylation status. Furthermore, H3K4 di-methylation may be associated with an open chromatin configuration and transcriptional activation (21). Aza increased the level of H3K4 di-methylation, however, there was no significant affect on $\mathrm{H} 3 \mathrm{~K} 9$ acetylation. TSA significantly increased the level of H3K9 acetylation in HEp-2 cells, although it produced a marginal effect on MGMT gene expression levels. The present study proposes that DNA methylation may be important in gene silencing and the maintenance of repressive histone modifications at hypermethylated gene promoters in laryngeal carcinogenesis. It has previously been reported that DNA modification itself, or components of the DNA methylation machinery, such as DNMTs or methyl CpG-binding proteins, may directly interact with histone methyltransferases or proteins in regions of DNA methylation. This interaction allows the DNA methylation machinery to assemble an alterative histone modification, demonstrating that histone methylation depends on DNA methylation $(22,23)$. 
The DNA methylation status of a gene has previously been reported as a promising biomarker in the early diagnosis and prognosis of cancer $(24,25)$. Aberrant DNA hypermethylation of gene promoter regions is an important epigenetic mechanism that regulates gene expression levels, resulting in the downregulation and silencing of various TSGs (26-28). In the present report, the MGMT gene was identified to exhibit a frequent methylation rate in LSCC, which may indicate that the occurrence of laryngeal cancer is associated with promoter methylation of the MGMT gene. The MGMT mRNA expression level is significantly lower when the promoter is methylated compared with when the promoter is unmethylated. Therefore, the mRNA expression level of the TSG, MGMT exhibits a negative correlation with CpG island methylation in LSCC. Furthermore, the MGMT methylation status exhibits no correlation with patient age or gender, tumor differentiation degree, tumor $\mathrm{T}$ stage or lymph node metastasis in LSCC. This lack of correlation indicates that MGMT gene methylation is not limited to a particular stage or sub-type of LSCC, but is involved in the entire development process. In addition, the present study examined the effect of Aza application on the migration and invasion ability of HEp-2 cells using wound-healing and Matrigel ${ }^{\circledR}$ invasion assays. Aza application inhibited the migration and invasion ability of HEp-2 cells, indicating that DNA demethylation may inhibit the invasion ability of HEp-2 cells. Recently, Aza was demonstrated to synergize with progesterone therapy to inhibit endometrial cancer cell growth and invasion (29). Thus, additional studies are required to fully elucidate the potential of epigenetic agents in cancer therapy.

In conclusion, the present study demonstrates that frequent epigenetic alterations regulate MGMT gene expression level in LSCC. The data provides a foundation for further investigations into the role of the MGMT gene in laryngeal carcinoma and its potential as a biomarker in the early diagnosis, treatment and prognosis of laryngeal carcinoma.

\section{References}

1. Marioni G, Marchese-Ragona R, Cartei G, Marchese F and Staffieri A: Current opinion in diagnosis and treatment of laryngeal carcinoma. Cancer Treat Rev 32: 504-515, 2006.

2. Bugni JM, Meira LB and Samson LD: Alkylation-induced colon tumorigenesis in mice deficient in the Mgmt and Msh6 proteins. Oncogene 28: 734-741, 2009.

3. Meng CF,Zhu XJ,Peng G and Dai DQ: Role of histone modifications and DNA methylation in the regulation of O6-methylguanine-DNA methyltransferase gene expression in human stomach cancer cells. Cancer Invest 28: 331-339, 2010.

4. McCabe MT, Brandes JC and Vertino PM: Cancer DNA methylation: molecular mechanisms and clinical implications. Clin Cancer Res 15: 3927-3937, 2009.

5. Kim YJ, Yoon HY, Kim SK, et al: EFEMP1 as a novel DNA methylation marker for prostate cancer: array-based DNA methylation and expression profiling. Clin Cancer Res 17: 4523-4530, 2011.

6. Chang X, Li Z, Ma J, et al: DNA methylation of NDRG2 in gastric cancer and its clinical significance. Dig Dis Sci 58: 715-723, 2013.

7. Wozniak RJ, Klimecki WT, Lau SS, Feinstein Y and Futscher BW: 5-Aza-2'-deoxycytidine-mediated reductions in G9A histone methyltransferase and histone $\mathrm{H} 3 \mathrm{~K} 9$ di-methylation levels are linked to tumor suppressor gene reactivation. Oncogene 26: 77-90, 2007.
8. Sharma S, Kelly TK and Jones PA: Epigenetics in cancer. Carcinogenesis 31: 27-36, 2010.

9. O'Sullivan B and Shah J: New TNM staging criteria for head and neck tumors. Semin Surg Oncol 21: 30-42, 2003.

10. Samudio-Ruiz SL and Hudson LG: Increased DNA methyltransferase activity and DNA methylation following epidermal growth factor stimulation in ovarian cancer cells. Epigenetics 7: 216-224, 2012.

11. Kondo Y, Shen L, Cheng AS, et al: Gene silencing in cancer by histone H3 lysine 27 trimethylation independent of promoter DNA methylation. Nat Genet 40: 741-750, 2008.

12. Socha MJ, Said N, Dai Y, et al:Aberrant promoter methylation of SPARC in ovarian cancer. Neoplasia 11: 126-135, 2009.

13. Wu LP, Wang X, Li L, et al: Histone deacetylase inhibitor depsipeptide activates silenced genes through decreasing both $\mathrm{CpG}$ and $\mathrm{H} 3 \mathrm{~K} 9$ methylation on the promoter. Mol Cell Biol 28: 3219-3235, 2008.

14. Grewal SI and Moazed D: Heterochromatin and epigenetic control of gene expression. Science 301: 798-802, 2003.

15. Chang X, Zhang S, Ma J, et al: Association of NDRG1 gene promoter methylation with reduced NDRG1 expression in gastric cancer cells and tissue specimens. Cell Biochem Biophys 66: 93-101, 2013.

16. Agathanggelou A, Cooper WN and Latif F: Role of the Ras-association domain family 1 tumor suppressor gene in human cancers. Cancer Res 65: 3497-3508, 2005.

17. Fahrner, JA, Eguchi S, Herman JG and Baylin SB: Dependence of histone modifications and gene expression on DNA hypermethylation in cancer. Cancer Res 62: 7213-7218, 2002.

18. Liu J, Zhu X, Xu X and Dai D: DNA promoter and histone $\mathrm{H} 3$ methylation downregulate NGX6 in gastric cancer cells. Med Oncol 31: 817, 2014.

19. Dong W, Wang L and Shen R: MYO5B is epigenetically silenced and associated with MET signaling in human gastric cancer. Dig Dis Sci 58: 2038-2045, 2013.

20. Chakraborty AK, Sousa Jde F, Chakraborty D, et al: GnT-V expression and metastatic phenotypes in macrophage-melanoma fusion hybrids is down-regulated by 5-Aza-dC: evidence for methylation sensitive, extragenic regulation of GnT-V transcription. Gene 374: 166-173, 2006.

21. Peng DF, Razvi M, Chen H, et al: DNA hypermethylation regulates the expression of members of the Mu-class glutathione S-transferases and glutathione peroxidases in Barrett's adenocarcinoma. Gut 58: 5-15, 2009.

22. Fuks F, Hurd PJ, Deplus R and Kouzarides T: The DNA methyltransferases associate with HP1 and the SUV39H1 histone methyltransferase. Nucleic Acids Res 31: 2305-2312, 2003.

23. Lin W and Dent SY: Functions of histone-modifying enzymes in development. Curr Opin Genet Dev 16: 137-142, 2006.

24. Kondo Y: Epigenetic cross-talk between DNA methylation and histone modifications in human cancers. Yonsei Med J 50: 455-463, 2009.

25. Schneider BG, Peng DF, Camargo MC, et al: Promoter DNA hypermethylation in gastric biopsies from subjects at high and low risk for gastric cancer. Int J Cancer 127: 2588-2597, 2010.

26. Tost J: DNA methylation: an introduction to the biology and the disease-associated changes of a promising biomarker. Methods Mol Biol 507: 3-20, 2009.

27. Tong JD, Jiao NL, Wang YX, Zhang YW and Han F: Downregulation of fibulin-3 gene by promoter methylation in colorectal cancer predicts adverse prognosis. Neoplasma 58: 441-448, 2011.

28. Zhi Y, Chen J, Zhang S, et al: Down-regulation of CXCL12 by DNA hypermethylation and its involvement in gastric cancer metastatic progression. Dig Dis Sci 57: 650-659, 2012.

29. Hu Q, Yu L, Chen R, Wang YL, et al: 5-aza-2'-deoxycytidine improves the sensitivity of endometrial cancer cells to progesterone therapy. Int J Gynecol Cancer 22: 951-959, 2012. 